\title{
Knowledge, Attitude and Practices Regarding HPV Vaccination Among Medical and Para Medical in Students, India a Cross Sectional Study
}

\author{
K Swarnapriya ${ }^{1 *}$, D Kavitha ${ }^{1 \&}$, Gopireddy Murali Mohan Reddy ${ }^{2}$
}

\begin{abstract}
Background: High risk human papilloma virus (HPV) types 16 and 18 have been proven as central causes of cervical cancer and safety and immunogenicity of HPV vaccines are sufficiently established. Knowledge and practices of HPV vaccination among medical and paramedical students is vital as these may strongly determine intention to recommend vaccination to others in the future. The present study was therefore undertaken to assess the knowledge, attitude and practices regarding cervical cancer screening and HPV vaccination among medical and paramedical students and to analyze factors influencing them. Materials and Methods: The present cross sectional study, conducted in a tertiary care teaching hospital in south India, included undergraduate students aged 18 years and above, belonging to medical, dental and nursing streams, after informed written consent. Results: Out of 957 participants, only $430(44.9 \%)$ displayed good knowledge and only $65(6.8 \%)$ had received HPV vaccination. Among the unvaccinated, $433(48.54 \%)$, were not willing to take the vaccine. Concerns regarding the efficacy $(30.5 \%)$, safety $(26.1 \%)$ and cost of the vaccine $(21.7 \%)$ were responsible for this. Age, gender, family history of malignancy and mother's education had no influence on knowledge. Compared to medical students, nursing students had better knowledge $(\mathrm{OR}-1.49,95 \%$ CI 0.96 to $2.3, \mathrm{p}=0.072)$ and students of dentistry had poor knowledge (OR-0.50 95\% CI 0.36 to $0.70, \mathrm{p}<0.001)$. Conclusions: The knowledge and uptake of HPV vaccination among medical and paramedical students in India is poor. Targeted health education interventions may have huge positive impact not only on the acceptance of vaccination among them, but also on their intention to recommend the vaccine in future.
\end{abstract}

Keywords: HPV vaccine - knowledge - attitude - practices - medical and paramedical students - India

Asian Pac J Cancer Prev, 16 (18), 8473-8477

\section{Introduction}

Cervical cancer is the most common cancer among women aged 15 years or older in India, with huge adverse social and economic impact on the families. Nearly 74,000 new cases of cervical cancer in India in 2010. Women in the reproductive age group (15-49 years) constituted $38 \%$ of these cases, indicating involvement of relatively younger population. In 1980, the mortality rate among incident cases of cervical cancer was 37 per 1000 new cases and there was little improvement in two decades as, the morality rate was 32 for every 100 new cases in 2010. (IHME_ChallengeAhead_FullReport; Forouzanfar et al., 2011) High mortality rates are largely attributed to diagnosis of the disease in advanced stages in majority of the affected patients. According to a study nearly $70 \%$ of cervical cancer cases in India are being diagnosed at an advanced stage (stage III or IV) (Vallikad, 2006).

High risk human papilloma virus (HPV) types (HPV 16 and 18) have been proved as not only the central cause of cervical cancer but also a necessary cause by studies conducted across the globe. Hence there is strong epidemiological evidence on the role of HPV in cervical cancer (Munoz, 2000). Many clinical trials and systematic reviews across the globe have reported the efficacy of vaccines against high risk HPV types (HPV 16 and 18) to be ranging from 90 to $99 \%$, in preventing cervical intraepithelial neoplasia (CIN). The safety of these vaccines is also sufficiently established by these reviews. (Araujo et al., 2013; Delere et al., 2014; Apter et al., 2015; Bruni et al., 2015; Kumar et al., 2015; Nakalembe et al., 2015). A recent review also reported the safety and Immunogenicity of HPV vaccines in Low and Middle income countries (LMIC) to be comparable to that of high income countries( HIC) (Nakalembe et al., 2015). Many recent studies published in India have also recommended the use of HPV based screening methods in the place of routine cytology based screening methods. (Sankaranarayanan et al., 2008; Suba et al., 2009; Basu et al., 2013).

${ }^{1}$ Department of Obstetrics and Gynaecology, ${ }^{2}$ Department of Community Medicine, Chettinad Hospital and Research Institute, Chennai, India *For correspondence: dr.swarnapriya@yahoo.com 
In spite of good understanding of the role of HPV in cervical cancer and well established efficacy of HPV based screening and vaccination as effective modes of primary prevention of carcinoma cervix, the uptake of these services is very poor in many developing countries, including India. (Raychaudhuri and Mandal, 2012a; Raychaudhuri and Mandal, 2012b). Lack of appropriate knowledge, negative attitude towards HPV vaccination and screening is considered as one of the most important factor for poor acceptance of these services by various studies. (Sankaranarayanan et al., 2003; Saha et al., 2010; Aswathy et al., 2012; Swapnajaswanth et al., 2014). Studies have also documented the intention to recommend these interventions are dependent heavily on the knowledge and perceptions of the Health care providers. (Riedesel et al., 2005; Daley et al., 2010).

Medical and paramedical students are future health care providers and can play a vital role in promoting cervical cancer screening and HPV vaccination. Importance of the right knowledge, positive attitude and high acceptance of these interventions among medical and paramedical students can't be undermined. Considering the paucity of studies in this regard, the present study has been undertaken with the following objectives.

To assess the knowledge, attitude and practices regarding cervical cancer screening and HPV vaccination among medical and paramedical students.To analyze the factors influencing the knowledge about HPV vaccination among the medical and paramedical students.

\section{Materials and Methods}

Study design: The study was a cross sectional study.

Study setting: The study was conducted in a tertiary care teaching hospital of a university in south India, where students from medical and various paramedical streams are being trained.

Study period: Data was collected over 2 month's period between May 2014 to July 2014.

Study subjects: The undergraduate students belonging to three streams including medical, dental and nursing colleges were included in the study.

Inclusion criteria: All the participants above 18 years of age.

Exclusion criteria: Women already diagnosed and treated for advanced stages of ca cervix; Women below 18 years.

Sample size and sampling method: Considering the lowest proportion of any particular KAP factor to be detected in study population as $10 \%$, with $95 \%$ power of study and an alpha error of 0.05 , the required sample size was 139 . To account for non participation, a minimum of 150 subjects were needed to be included from each of the study groups. But all the available students at the time of data collection were included in the study.

Sampling method: All the eligible subjects in the study setting were recruited in to the study, hence no sampling was done.

Study tools: A standardized questionnaire, developed for the purpose of study was used. The questionnaire was validated by distributing it to five experts in the field and getting their consensus on each item. Pilot testing was done to further validate the questionnaire and to assess the feasibility of administration of the questionnaire and necessary changes were done.

Ethical considerations: Ethical approval of Institutional Human ethical committee was obtained. For all the eligible study subjects, informed written consent was obtained after thoroughly explaining purpose and nature of study. For those who refuse to give consent, reasons for the same were documented.

Statistical analysis: The current status of knowledge, vaccination status and willingness to vaccinate were the primary outcome measures. Various socio demographic variables, course and year of study were the explanatory variables. Descriptive analysis of all the explanatory and outcome variables was done by using mean and standard deviation for quantitative variables, frequency and percentages for categorical variables. The association between the explanatory variables and knowledge was assessed by doing multivariate logistic regression analysis, after including apprpropriate variables in the model. The adjusted odds ratio, 95\% confidence intervals and p-values are presented. $\mathrm{P}$ value less than 0.05 was considered as statistically significant. Microsoft Excel and IBM SPSS were used for analysis.

\section{Results}

A total of 957 participants were interviewed in the study. The mean age of the participants was $19.25( \pm 1.64)$ years and Majority (71.6\%) of the participants was females. The proportion of study participants from each of the four study years was almost similar. The assessment of educational status of the mother showed, 250 (26.2\%) mothers completed secondary schooling, the number of women who completed graduation and post graduation

Table 1. Descriptive Analysis of Socio Demographic Variables (N=957)

\begin{tabular}{lcc}
\hline Parameter & Frequency & Percent \\
\hline I. Sex & & \\
Male & 272 & 28.4 \\
Female & 685 & 71.6 \\
II. Course & & \\
BDS & 290 & 30.3 \\
MBBS & 476 & 49.7 \\
Nursing & 191 & 20 \\
III. Year of study & & \\
1 & 273 & 28.5 \\
2 & 236 & 24.7 \\
3 & 225 & 23.5 \\
4 & 223 & 23.3 \\
VII. Mothers education & & \\
Illiterate & 40 & 4.2 \\
Primary school & 174 & 18.2 \\
Secondary school & 250 & 26.1 \\
Graduation & 307 & 32.1 \\
Post Graduation & 186 & 19.4 \\
XIII. Family History of Malignancy & & \\
Yes & 75 & 7.8 \\
No & 882 & 92.1 \\
Age (Mean +_SD) & & $19.25 \pm 1.64$ \\
\hline
\end{tabular}



Table 2. Descriptive Analysis of Knowledge about HPV Vaccine in Students (N=957)

\begin{tabular}{|c|c|c|}
\hline Knowledge related to HPV vaccineParameter & Frequency & $\%$ \\
\hline I know about HPV vaccine & 827 & 86.4 \\
\hline People correctly expanded HPV & 676 & 70.6 \\
\hline \multicolumn{3}{|l|}{ HPV vaccination provides protection against } \\
\hline Carcinoma Cervix & 514 & 60.1 \\
\hline Laryngeal papilloma & 195 & 20.1 \\
\hline Genital Warts & 154 & 15.9 \\
\hline Carcinoma Penis & 86 & 8.8 \\
\hline Three doses of vaccine should be given & 394 & 41.2 \\
\hline HPV Administered through IM & 553 & 57.7 \\
\hline HPV Administered at $10-25$ years & 498 & 52 \\
\hline HPV Administered in Males also & 425 & 44.4 \\
\hline HPV Efficacy is $>90 \%$ & 171 & 17.9 \\
\hline Pregnancy is a Contradiction for HPV vaccination & 464 & 48.4 \\
\hline HPV can be Administered to already infected people & 301 & 31.5 \\
\hline Having overall good knowledge about HPV vaccination (answering 6 or more questions correctly) & 430 & 44.9 \\
\hline
\end{tabular}

Table 3. Reported Source of Information about HPV Vaccination

\begin{tabular}{lcr}
\hline Source of Knowledge for HPV & Frequency & $\%$ \\
\hline Teacher & 652 & 68.1 \\
Internet & 119 & 12.4 \\
TV & 90 & 9.4 \\
Magazine & 46 & 4.6 \\
Others & 92 & 9.6 \\
\hline
\end{tabular}

were $307(32.1 \%)$ and $186(19.4 \%)$ respectively. There were only $40(4.2 \%)$ of the mothers, who were illiterates and remaining $174(18.2 \%)$ have completed primary schooling. It can be concluded that the majority of the study participant's mothers were educated beyond secondary schooling. Seventy five $(7.8 \%)$ had reported family history of malignancy, but the type and part of the body affected were not explored in the study. (Table 1)

Even though high proportion of students has reported that they know about the HPV, only $70.6 \%$ could expand it correctly. Only $514(60.1 \%)$ participants are aware that HPV can prevent carcinoma cervix. The proportion of participants who are aware about the role of HPV in preventing other diseases like laryngeal papilloma, genital warts and carcinoma penis was very small ranging from 8 to $20 \%$. The proportion of subjects who displayed correct knowledge about dose, route and age of administration was ranging from 41.2 to $57.7 \%$. Only 171 (17.9\%) of the participants felt that the vaccine had more than $95 \%$ efficacy. The proportion of subjects, who reported that the vaccine can be given to males, was $44 \%$. The people who reported pregnancy as contraindication for vaccine was $48.4 \%$ and $31.5 \%$ of the subjects felt that the vaccine can be given even after the person is infected with HPV. Overall only $430(44.9 \%)$ of the participants displayed good knowledge regarding HPV vaccination. (Table 2)

The most commonly reported source of information about HPV was the teacher, which was reported by 652 $(68.1 \%)$ of the participants. The other reported sources of information were internet (12.4\%), television (9.4\%) and magazines $(4.6 \%)$ (Table 3$)$.

The number of subjects, who have already received HPV vaccination were only 65(6.8\%) among the study group. Out of the 892 subjects, who did not receive the
Table 4. Descriptive Analysis of Practices about HPV Vaccine in Students ( $\mathbf{N = 9 5 7 )}$

\begin{tabular}{lrc}
\hline ParameterParameter & Frequency & $\%$ \\
\hline HPV Immunized & 65 & 6.8 \\
Yes & 892 & 93.2 \\
No & \multicolumn{2}{c}{} \\
Now willing to take HPV (N=892) & 459 & 51.46 \\
Yes & 433 & 48.54 \\
No & 132 & 30.48 \\
Reason for not willing among unwilling people $(\mathrm{N}=433)$ \\
Doubts regarding efficacy of the vaccine & 113 & 26.1 \\
Fear of side effects & 94 & 21.71 \\
Cost & 63 & 14.55 \\
Minimal risk of carcinoma cervix & 31 & 7.16 \\
No response & \multicolumn{2}{l}{} \\
\hline
\end{tabular}

Table 5. Multivariate Logistic Regression Analysis of Factors Influencing the Knowledge Regarding HPV (N=957)

\begin{tabular}{lclll}
\hline Parameter & $\begin{array}{c}\text { Adjusted } \\
\text { odds }\end{array}$ & \multicolumn{2}{c}{$\begin{array}{c}\text { 95\% C.I. for } \\
\text { odds ratio }\end{array}$} & P-value \\
\cline { 3 - 4 } & ratio & Lower & Upper & \\
\hline Age & 1.067 & 0.943 & 1.208 & 0.3 \\
Gender (Male Vs female) & 0.769 & 0.56 & 1.055 & 0.1 \\
Family History of malignancy & 1.058 & 0.643 & 1.742 & 0.82 \\
Course (MBBS- Baseline) & & & & \\
BDS & 0.504 & 0.363 & 0.701 & $<.001$ \\
Nursing & 1.497 & 0.965 & 2.323 & 0.072 \\
Year of study (First Year - Baseline) & & & \\
Second year & 1.332 & 0.889 & 1.997 & 0.16 \\
Third year & 2.539 & 1.596 & 4.04 & $<.001$ \\
Final Year & 3.614 & 2.055 & 6.356 & $<.001$ \\
Mother's education (Illiterate-Baseline) & & & \\
Primary schooling & 0.621 & 0.297 & 1.3 & 0.2 \\
Secondary schooling & 0.588 & 0.272 & 1.268 & 0.17 \\
Graduate & 0.642 & 0.297 & 1.386 & 0.26 \\
Post graduate/Professional & 0.761 & 0.341 & 1.7 & 0.5 \\
\hline
\end{tabular}

vaccine, 459 (51.46) subjects reported that they are willing to take HPV vaccine and the remaining 433(48.54\%) were not willing to receive the vaccine. The most common reasons for the negative attitude were doubts regarding the efficacy of the vaccine (30.48\%), fear of side effects $(26.10 \%)$ and prohibitive cost of the vaccine $(21.71 \%)$. 
Lower perceived risk of Carcinoma cervix was the reason in $63(14.55 \%)$ participants and $31(7.16 \%)$ of the participants did not respond to the question. (Table 4).

Age of the participant, gender and family history of malignancy had no significant influence on the knowledge about HPV vaccination. Compared to MBBS students, the BDS students had poor knowledge (OR-0.50 95\%CI 0.36 to 0.70 , p value $<0.001$ ) about HPV. Even though Nursing students had better knowledge compared to MBBS students (OR-1.49, 95\% CI 0.96 to 2.3, $\mathrm{p}=0.072$ ), it was not statistically significant. With increasing seniority the odds of good knowledge about HPV has increased. Compared to first year students, the odds of good knowledge was 1.33 (95\%CI 0.88 to 1.99 , p value 0.16 ), $2.53(95 \% \mathrm{CI} 1.59$ to 4.04 , $\mathrm{p}$ value $<0.01)$ and 3.61(95\% CI 2.05 to $3.65, \mathrm{p}$ value $<0.001)$. Mother's education had no association with knowledge level (Table 5).

\section{Discussion}

HPV vaccination alone or combined with screening have been documented as effective interventions in reducing the burden and mortality due to carcinoma cervix across various settings. Many studies conducted in India have documented the prevalence of high risk HPV infections .i.e. HPV $16 / 18$ to be ranging from about $30 \%$ in inflammatory lesions to more than $95 \%$ in cases of cervical cancer. (Bhatla et al., 2008; Basu et al., 2009; Deodhar et al., 2012; Basu et al., 2013). Many authors have also suggested that achieving high levels of HPV 16/18 vaccination would have a significant health and economic impact by reducing the burden of cervical cancer. (Armstrong, 2010; Deodhar et al., 2012; Nakalembe et al., 2015).

In spite of the proven efficacy and potential for huge positive impact at the community level, the uptake of HPV vaccination is very low in many developing countries, including India. There are many reasons like, lower perceived risk of cervical cancer, non availability of vaccine, prohibitive cost (Das et al., 2008) etc. But lack of appropriate knowledge about role of HPV in causation of carcinoma cervix and HPV vaccine is one of the most important determining factors for poor uptake of vaccination. (Barnack et al., 2010).

In the current study, only $44.9 \%$ of the participants displayed good knowledge regarding HPV vaccination. The most striking aspect of the study is only $17.9 \%$ of the subjects believed that vaccine had more than $95 \%$ efficacy. The overall knowledge about the dosage, route, site of the vaccine and it's role in preventing conditions other than carcinoma cervix, especially in males was poor in the study.

The poor knowledge has reflected in poor uptake of vaccination, as only $6.8 \%$ have already received HPV vaccination. Out of the non vaccinated people, $48.54 \%$ don't want to vaccinate themselves. The reported reasons for the same were doubts regarding the efficacy of the vaccine $(30.48 \%)$, fear of side effects $(26.10 \%)$, prohibitive cost $(21.71 \%)$ and lower perceived risk of Carcinoma cervix (14.55\%).

Knowledge regarding HPV, HPV vaccination, cervical screening and cervical cancer risk factors was remarkably poor in various population groups like general population, university students, health care staff etc. (Makwe et al., 2012). Intention to receive an HPV vaccine was significantly associated with knowledge. Providing education about the etiology of cervical cancer and the HPV link is an essential component to enhance HPV vaccine uptake. (Wong and Sam, 2010).

Many studies have demonstrated, the proportion of subjects with intention to vaccinate themselves or their kids ranging from about $70 \%$ to $100 \%$ once they are educated about the vaccine. (Francis et al., 2010; Poole et al., 2013).The acceptance rates are very high even in men after proper education. (Belani et al., 2014).

Knowledge in medical and paramedical personnel: Knowledge among health care providers is even more important, as it may influence their intention to recommend the vaccine (Riedesel et al., 2005) and in turn can affect overall vaccine uptake in the community (Daley et al., 2010).

Some studies have documented that, the knowledge regarding the causal relationship between HPV and cervical cancer were much higher in medical and paramedical students, compared to other students. But the difference in the proportion of people who are willing to get vaccinated was not very striking between these groups (Uzunlar et al., 2013). The factors influencing this mismatch between knowledge and willingness to vaccinate were not clearly explored by these studies.

Many studies conducted among physicians (Wong et al., 2013) and nurses have documented that, the most important factors considered by health care practitioners while recommending HPV vaccine included effectiveness, side effects/safety. Clear recommendations by the Government and cost were also considered important. Many of these studies emphasized the importance of more education about the virus, cervical cancer and the vaccine are required to further increase the willingness to recommend the vaccine (Wamai et al., 2013). Educational initiatives targeting health care practitioners are strongly recommended along with initiatives to reduce the cost and clear guidelines by governments, to achieve immunization rates in the given communities (Wong et al., 2013). Government-sponsored or school-based programmes were also recommended as initiatives which may improve HPV-related knowledge and HPV vaccine acceptability. (Chang et al., 2013).

In conclusion, the knowledge regarding HPV vaccination among medical and paramedical students is poor. The nursing students displayed better knowledge compared to MBBS and dental students. the HPV vaccine uptake is also very poor among medical and paramedical students. among unvaccinated, more than 50\% are not willing to take vaccine, the main reason being concerns about efficacy and safety of the vaccine. Lower perceived severity of illness is also another important reason.

\section{References}

Apter D, Wheeler CM, Paavonen J, et al (2015). Efficacy of human papillomavirus 16 and 18 (HPV-16/18) AS04- 
Knowledge, Attitude and Practices Regarding HPV Vaccination among Medical and Para Medical Students in India adjuvanted vaccine against cervical infection and precancer in young women: final event-driven analysis of the randomized, double-blind PATRICIA trial. Clin Vaccine Immunol, 22, 361-73.

Araujo SC, Caetano R, Braga JU, et al (2013). Efficacy of commercially available vaccines against HPV infection in women: a systematic review and meta-analysis. Cad Saude Publica, 29, 32-44.

Armstrong EP (2010). Prophylaxis of cervical cancer and related cervical disease: a review of the cost-effectiveness of vaccination against oncogenic HPV types. J Manag Care Pharm, 16, 217-30.

Aswathy S, Quereshi MA, Kurian B, et al (2012). Cervical cancer screening: Current knowledge \& practice among women in a rural population of Kerala, India. Indian J Med Res, 136, 205-10.

Barnack JL, Reddy DM, Swain C (2010). Predictors of parents' willingness to vaccinate for human papillomavirus and physicians' intentions to recommend the vaccine. Womens Health Issues, 20, 28-34.

Basu P, Mittal S, Bhaumik S, et al (2013). Prevalence of high-risk human papillomavirus and cervical intraepithelial neoplasias in a previously unscreened population--a pooled analysis from three studies. Int J Cancer, 132, 1693-9.

Basu P, Roychowdhury S, Bafna UD, et al (2009). Human papillomavirus genotype distribution in cervical cancer in India: results from a multi-center study. Asian Pac J Cancer Prev, 10, 27-34.

Belani HK, Sekar P, Guhaniyogi R, et al (2014). Human papillomavirus vaccine acceptance among young men in Bangalore, India. Int J Dermatol, 53, 486-91.

Bhatla N, Dar L, Rajkumar Patro A, et al (2008). Human papillomavirus-type distribution in women with and without cervical neoplasia in north India. Int J Gynecol Pathol, 27, 426-30.

Bruni L, Serrano B, Bosch X, et al (2015). Human papillomavirus vaccine. Efficacy and safety. Enferm Infecc Microbiol Clin, 33, 342-54.

Chang IJ, Huang R, He W, et al (2013). Effect of an educational intervention on HPV knowledge and vaccine attitudes among urban employed women and female undergraduate students in China: a cross-sectional study. BMC Public Health, 13, 916.

Daley EM, Vamos CA, Buhi ER, et al (2010). Influences on human papillomavirus vaccination status among female college students. J Womens Health (Larchmt), 19, 1885-91.

Das BC, Hussain S, Nasare V, et al (2008). Prospects and prejudices of human papillomavirus vaccines in India. Vaccine, 26, 2669-79.

Delere Y, Wichmann O, Klug SJ, et al (2014). The efficacy and duration of vaccine protection against human papillomavirus: a systematic review and meta-analysis. Dtsch Arztebl Int, 111, 584-91.

Deodhar K, Gheit T, Vaccarella S, et al (2012). Prevalence of human papillomavirus types in cervical lesions from women in rural Western India. J Med Virol, 84, 1054-60.

Forouzanfar MH, Foreman KJ, Delossantos AM, et al (2011). Breast and cervical cancer in 187 countries between 1980 and 2010: a systematic analysis. Lancet, 378, 1461-84.

Francis SA, Nelson J, Liverpool J, et al (2010). Examining attitudes and knowledge about HPV and cervical cancer risk among female clinic attendees in Johannesburg, South Africa. Vaccine, 28, 8026-32.

Kumar S, Biswas M, Jose T (2015). HPV vaccine: Current status and future directions. Med J Armed Forces India, 71, 171-7.

Makwe CC, Anorlu RI, Odeyemi KA (2012). Human papillomavirus (HPV) infection and vaccines: knowledge, 